\title{
Towards a novel therapy against AIDS
}

\author{
Adrien Locatelli \\ Lycée les Eaux Claires, 1 rue des Eaux Claires, 38100 Grenoble, France
}

\section{A B S T R A C T}

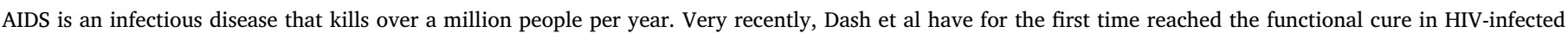

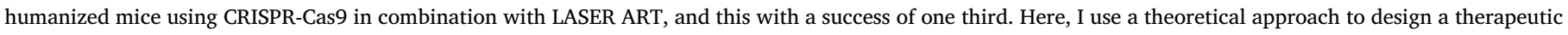

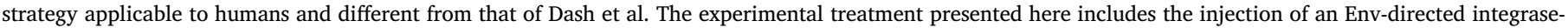

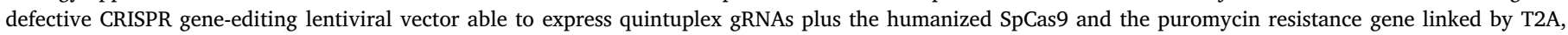

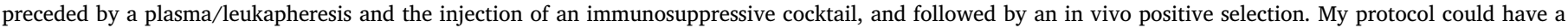

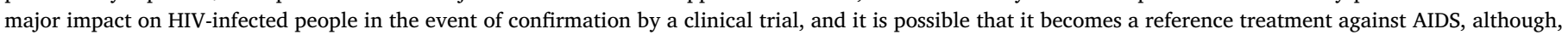
for the moment, it is only at the stage of hypothesis and theory.

\section{Background}

Acquired Immune Deficiency Syndrome (AIDS) is an infectious disease that kills over a million people per year and remains a major public health concern that affects over 35 million people worldwide. Since the early 1980s, it is known that AIDS is caused by the human immunodeficiency virus (HIV), which has the particularity of being a retrovirus of the lentivirus family [1]. The number of new HIV infections is estimated at more than five thousand per day. Antiretroviral therapy (ART) effectively suppress HIV replication by blocking various steps of the viral life-cycle. However, ART does not succeed in eliminating integrated proviral DNA from the genome of infected cells [2-3]. As a result, HIV persists in a dormant state within infectious reservoirs, and ART cessation leads to viral reactivation and disease progression to AIDS [4]. Therefore, a major challenge for a curative strategy is to eliminate either integrated proviral DNA or infected cells. However, the elimination of HIV in humans is documented only in two patients [5-6].

Since the early 2010s, CRISPR associated protein 9 (Cas9) has greatly enhanced our ability to make genomic modifications, bringing a revolution in genome engineering, with the rapid development of new techniques. A very large number of studies has demonstrated the efficiency of this easy-to-use, flexible, and powerful tool [7-18]. In parallel, an equally large number of studies has shown that CRISPR-Cas9based gene-editing technology can durably inhibit HIV replication by specifically excising fragments of integrated proviral DNA from the genome of infected cells [19-30]. More particularly, it has been shown that single gRNA CRISPR therapy results in hypermutation of gRNA target sites but dual gRNA CRISPR therapy can functionally cure HIVinfected cells [28]. It has also been shown that CRISPR-Cas9 can remove integrated proviral DNA from the genomes of humanized mice [29]. And very recently, we were able to attend the very first permanent elimination of the virus in HIV-infected humanized mice [30]. By drastically reducing the size of the viral reservoir at an early stage of the infection using long-acting slow-effective release ART (LASER ART) and by excising HIV subgenomic DNA fragments using AAV9-mediated CRISPR-Cas9-based gene-editing technology, Dash et al have for the first time eliminated replication-competent HIV DNA from the genomes of living animals. A third of their mice that received sequential LASER ART and CRISPR-Cas9 therapy became virus-free (two mice on seven).

\section{Presentation of the hypothesis}

\section{Strategy development}

Foremost, although, for the moment, only the combination LASER ART plus CRISPR-Cas9 allowed the permanent elimination of the virus within the animal model, I have chosen not to explore the use of LASER ART in combination with the therapeutic strategy presented here because the use of LASER ART requires a very early management of the infection which could not be performed in all patients since no proof-ofconcept for LASER ART has yet been achieved in humans. However, LASER ART is very promising, and its action of reducing the size of the reservoir of infection could greatly improve the efficiency of the experimental treatment that I propose.

The high genetic variability of HIV is one of the first obstacles to the development of a curative strategy. Indeed, HIV differs from many viruses in that it has a very high genetic variability, which is the result of its fast replication cycle, with the generation of about $10^{10}$ virions per day, coupled with a high mutation rate of approximately $3 \times 10^{-5}$

E-mail address: adriloc38@gmail.com. 
Table 1

Selected gRNAs Targeting HIV-1.

\begin{tabular}{|c|c|c|c|c|}
\hline Name & $\begin{array}{l}\text { Position in HIV-1 } \\
\text { LAI DNA }^{\mathrm{a}}\end{array}$ & \multicolumn{2}{|c|}{ Target Sequence + PAM $^{\mathrm{b}}$} & Orientation \\
\hline gLTR7 & $\begin{array}{l}413-435 ; \\
9544-9566\end{array}$ & \multicolumn{2}{|c|}{ TGCTTATATGCAGCATCTGA(GGG) } & antisense \\
\hline gGag3 & $1480-1502$ & \multirow{4}{*}{\multicolumn{2}{|c|}{$\begin{array}{l}\text { CCTGCTATGTCACTTCCCCT(TGG) } \\
\text { GATGACAGCATGTCAGGGAG(TGG) } \\
\text { GTTGCCAAAGAGTGATTTGA(GGG) } \\
\text { GGAGCAGCAGGAAGCACTAT(GGG) }\end{array}$}} & antisense \\
\hline gGag4 & 1827-1849 & & & sense \\
\hline gGagPol & $2288-2310$ & & & antisense \\
\hline gEnv2 & $7841-7863$ & & & sense \\
\hline \multicolumn{2}{|c|}{ Specificity Score $(\%)^{\mathrm{c}}$} & Conservation $(\%)^{\mathrm{d}}$ & \multicolumn{2}{|c|}{ Corrected Conservation (\%) } \\
\hline 60 & & 46 & 98 & \\
\hline 67 & & 66 & 98 & \\
\hline 44 & & 57 & 98 & \\
\hline 66 & & 47 & 98 & \\
\hline 69 & & 74 & 98 & \\
\hline
\end{tabular}

(a) The genome of LAI isolate is a 9719-bp-long reference genome first sequenced by Barre-Sinoussi et al. [1]. (b) Protospacer-adjacent motif (PAM) sequence is in parentheses. (c) Specificity score was calculated using the algorithm developed by the Zhang laboratory (zlab.bio). (d) Conservation rate was calculated from the HIV database maintained by the Los Alamos National Laboratory (www.hiv.lanl.gov). (e) It has been shown that Streptococcus pyogenes Cas9 (SpCas9) tolerates a number of mismatches between gRNA and target DNA [13]. Therefore, the conservation rate is not representative of the efficiency of the gRNA. However, Shannon entropy is a good tool to measure representative conservation of the target sequence. That is why I calculated a representative corrected conservation rate equal to $\mathrm{e}^{\mathrm{W}(-\mathrm{S} / 4)}$ where $\mathrm{W}$ is the Lambert $\mathrm{W}_{0}$ function, and $\mathrm{S}$ is the Shannon entropy (The HIV database of the LANL use the natural logarithm and not the binary logarithm).

per nucleotide base per replication cycle and the recombinogenic properties of reverse transcriptase [31-33]. However, this problem can be solved by identifying and then targeting highly conserved sequences of the HIV genome. Thus, as seen in previous studies, the CRISPR design web tool developed by the Zhang laboratory (zlab.bio) was naturally used to select five gRNAs targeting highly conserved sequences of the sense or antisense strand of the HIV-1 M group DNA (subtype B LAI isolate) with a high specificity score ( $>40 \%$ ) (Table 1 ; Fig. 1). These five gRNAs are partially identical to five gRNAs previously identified by Wang et al and whose Shannon entropy is less than 0.1 (My gGagPol differs from one nucleotide) [28]. One gRNA targets the long-terminal repeat (LTR) sequences that are present at both the $5^{\prime}$ and $3^{\prime}$ end of the HIV DNA, and the remaining four gRNAs target sequences encoding proteins essential for viral replication. Furthermore, these five gRNAs have already been tested in vitro by Wang et al either alone or in combination, and no significant toxicity was detected.

After identifying five robust gRNAs targeting highly conserved sequences of the HIV-1 M group genome, I then proceeded to the design of the vector. As seen previously, Dash et al who are the first to have reached the functional cure in animals opted for an AAV9-mediated CRISPR therapy [30]. Although there is a multiplicity of vector systems for gene delivery, the two most commonly used vectors in the previous work on the subject were AAV and LV (Lentiviral Vector). Several reasons led me to choose the gene delivery mediated by LV rather than AAV. This mainly includes a larger cargo space and a more appropriate tropism. Thus, an all-in-one pLV able to deliver multiplex gRNAs plus SpCas9 was designed using SnapGene (Fig. 2; https://figshare.com/ articles/pLV/9642281/1) [34]. The model on which this pLV is based is the backbone of the "Mammalian Dual-gRNA Expression Lentiviral Vector" from VectorBuilder. First off, pLV[5CRISPR]-hCas9:T2A:PuroU6 $>$ gRNA carries the humanized SpCas9 and the puromycin resistance gene linked by T2A under the control of the EFS promoter in the form of an expression cassette. Then, of the five gRNAs previously identified and incorporated into cassettes under the U6 promoter, two were placed upstream and three downstream of the EFS $>$ hCas9:T2A:Puro expression cassette in order to prevent recombination. Still against recombination, a random DNA generator ( $40 \%$ GC-content) was used to generate relatively long separation sequences to space the downstream cassettes. In theory, the more different gRNAs expressed simultaneously there are, the more efficient the strategy should be. However, technical difficulties, including recombination problems and limited cargo space, did not allow me to exceed five gRNAs. Although I did everything I could to prevent problems due to recombination, perhaps that is not enough, perhaps it would be more judicious to use different alternative promoters and not to exceed four gRNAs. Only a series of in vitro tests would decide the question. Furthermore, the genome of pLV[5CRISPR]-hCas9:T2A:Puro-U6 > gRNA is 9718 bp long (one less than LAI isolate genome), which suggests that it is not oversized and its titer will not be affected.

I have just designed an all-in-one CRISPR gene-editing pLV, but there are still several problems with the use of a standard third-generation LV. Firstly, because of VSV-G pseudotyping, the ability of standard third-generation LV to transduce hematopoietic stem cells (HSCs) and peripheral blood mononuclear cells (PBMCs) remains very limited, while they are infectious reservoirs to be targeted as a priority in the context of the search for a functional cure. Indeed, HSCs and PBMCs lack the LDL receptor, which is required for entry and transduction of VSV-G pseudotyped LV [35]. However, this problem can be solved quite simply by returning to Env-directed lentiviral delivery. Furthermore, Env-directed LV has the same tropism as HIV, which is a positive point for transducing infected cells. Then, the permanent integration of the vector poses two problems, namely random transgenesis and time-induced exacerbation of off-target effects. However, the cause of these two other problems can be solved in an equally simple way by using an integrase-defective LV instead of a standard LV.

After completely finishing the design of the vector on which the whole strategy is based, I then proceeded to the design of the strategy itself. First and foremost, since LV is derived from HIV, it is also sensitive to ART. That is why the patient will have to stop any ART a few weeks before the start of the treatment and have no trace of it in his body throughout the duration of treatment to avoid any disruption. Then, when the body of the patient will be ART-free, and immediately before the intravenous injection of the vector, a leukapheresis procedure should remove a large part of its leukocytes including PBMCs to improve the multiplicity of infection. However, there is one last

\section{Sense gRNAs}

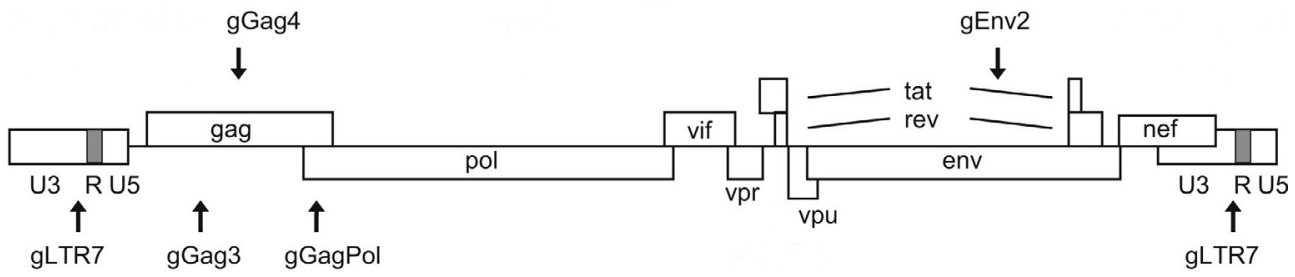

\section{Antisense gRNAs}

Fig. 1. Map of target sequences. 


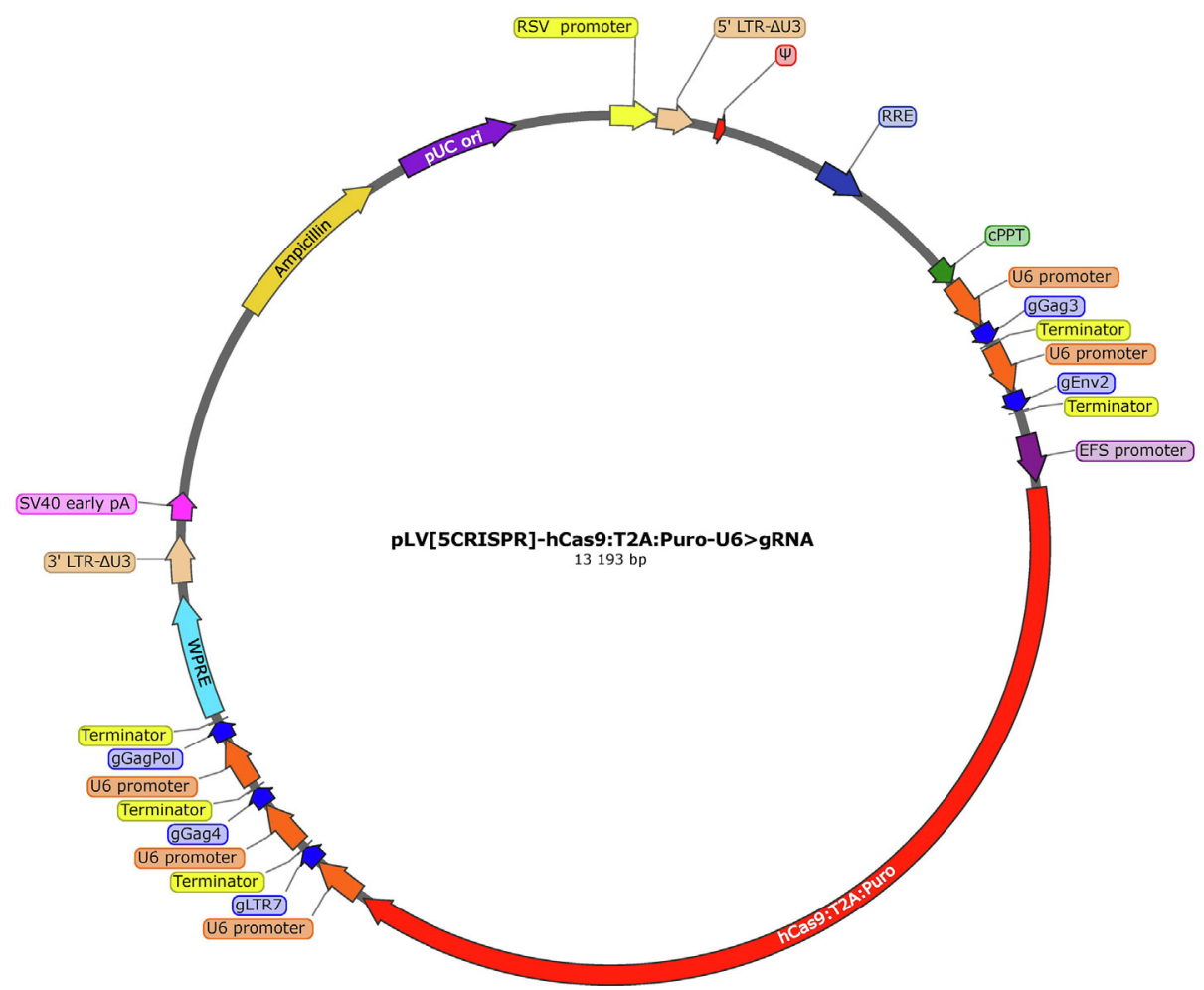

Fig. 2. Map of pLV[5CRISPR]-hCas9:T2A:Puro-U6 > gRNA (https://figshare.com/articles/pLV/9642281/1).

problem to be solved before the injection of the vector. Indeed, in vivo use of an Env-directed vector poses a problem because Env-directed LV is rapidly eliminated by the immune system. However, this other problem can be solved by coupling leukapheresis with plasmapheresis to eliminate most of the circulating Env-targeting antibodies and by injecting an immunosuppressive cocktail as in the case of autoimmune disease or transplant rejection. In addition, injection of the two subunits of the Env protein produced by genetic recombination would "lure" the few remaining antibodies. Then, knowing that the number of PBMCs is of the order of $3.5 \times 10^{9}$ per liter of blood and the number of HSCs is of the order of $10^{4}$ per human body [36-37], an intravenous injection of $5 \times 10^{11}$ transduction units of pLV[5CRISPR]-hCas9:T2A:Puro-U6 > gRNA should transduce a large part of the HIV-infected cells of the patient. With a titer of $10^{9} \mathrm{TU} / \mathrm{ml}$, a single intravenous injection of $500 \mathrm{ml}$ of a pLV[5CRISPR]-hCas9:T2A:Puro-U6 > gRNA-containing solution should be enough. Then, since pLV[5CRISPR]-hCas9:T2A:Puro-U6 > gRNA carries the puromycin resistance gene, it is possible to conduct a positive selection by intravenously injecting puromycin a few weeks after transduction. Positive selection is a technique that is widely used in vitro but very rarely in vivo. However, I think that it could become a powerful innovation in the medical fight against AIDS. Indeed, by considerably reducing the size of the viral reservoir, in vivo positive selection could solve the problem of the rapid spread of the infection by continuous sources of virus. As the target cells have a rather low replication rate, the non-integration of the vector is not an obstacle to selection. The key to a successful in vivo positive selection is to inject enough puromycin to affect the infectious reservoirs but not enough to harm the life of the patient. I think that it would be more appropriate to use a myristoylated prodrug rather than standard puromycin. Indeed, it would increase lipophilicity and would allow better absorption by bone marrow cells. The myristoylated puromycin dihydrochloride prodrug should be synthesizable without much technical difficulties according to the method described below (Fig. 3). A dosing schedule of $1.2 \mathrm{mg} / \mathrm{kg} /$ day intravenously during three series of three days with a seven-day spacing between each series should produce the expected effect with a mortality rate strictly less than $5 \%$. This dosage is based on that of melarsoprol but it has been readjusted according to the theorical intravenous $\mathrm{LD}_{50}$ of the prodrug and the desired effect. Furthermore, it has been shown that the presence of P-glycoprotein (P-gp) inhibitors considerably enhances the puromycine-mediated positive selection of LV-transduced HSCs [38]. Thus, the administration of a third-generation P-gp inhibitor should be performed in combination with the puromycin prodrug to boost the drug-selection. A dose of $4 \mathrm{mg} / \mathrm{kg} /$ day of tariquidar (only on days when the puromycin prodrug is also delivered) by intravenous route should be more than enough. Although the association Cas9 plus positive selection is very similar to an eradication cure, it can not be qualified as such. Indeed, even with this approach, total sterilization of all infectious reservoirs is impossible.

Summary of the protocol of the proposed therapeutic strategy

I hypothesize that it is possible to functionally cure an HIV-infected patient by applying the following experimental action plan:

1. Start stopping taking any ART and any substance that may have antiretroviral action a few weeks before the start of the actual treatment in order to remove any trace of ART from the body of the patient.

2. Once the body of the patient is ART-free, apply a total plasma/ leukapheresis procedure in order to remove as many leukocytes and antibodies as possible from the blood of the patient.

3. Then inject immediately after, by intravenous route, an immunosuppressive cocktail of the same type as those administered in the case of autoimmune disease or transplant rejection in order to minimize the action of the remaining immune system. If necessary, complete the cocktail with a protein suspension composed of the two subunits of the Env protein produced by genetic recombination having the function of "luring" the few remaining antibodies.

4. After waiting a few minutes for the cocktail to take effect and dissipate, inject, still by intravenous route, $500 \mathrm{ml}$ of a solution containing $5 \times 10^{11}$ transduction units of my Env-directed integrasedefective CRISPR gene-editing lentiviral vector (whose .dna file is available at https://figshare.com/articles/pLV/9642281/1), which 


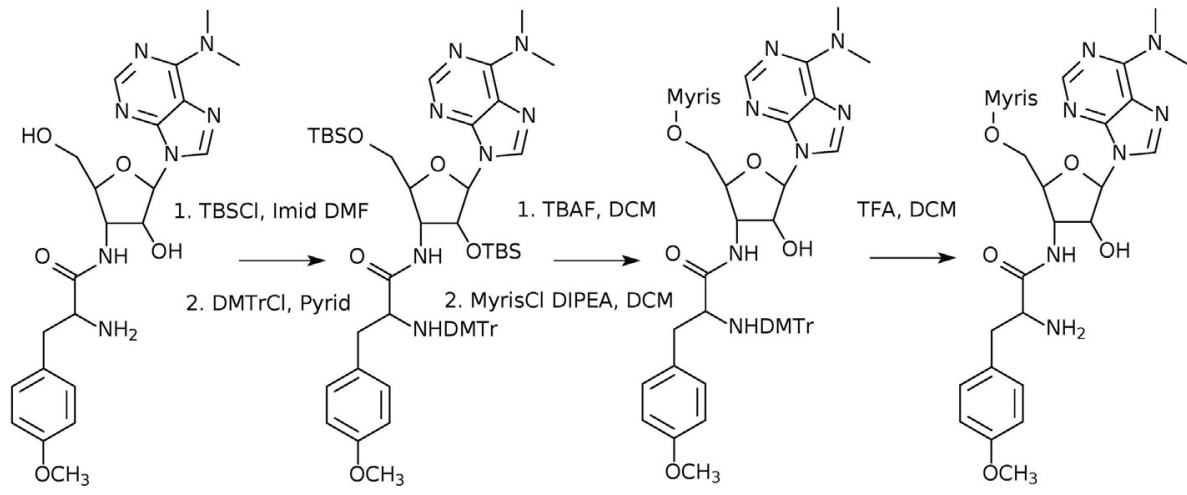

(1)
(3)

Fig. 3. Myristoylated puromycin prodrug synthesis. Freebase puromycin (1 equivalent) should be dried by azeotroping from anhydrous pyridine, then suspended in anhydrous dimethylformamide and cooled to $0{ }^{\circ} \mathrm{C}$ under inert gas. Selective silylation of the two hydroxyl groups should be conducted by adding tertButyldimethylsilyl chloride (2.4 equivalents) and imidazole ( 3 equivalents) to the suspension. The amino group in the silylated puromycin should then be protected by reacting with dimethoxytrityl chloride (2.2 equivalents) in anhydrous pyridine to orthogonally protect the molecule. Selective cleavage of the silyl groups should be achieved using tetra-nbutylammonium fluoride (5 equivalents) in dichloromethane to unlock the two hydroxyl groups. The primary alcohol should then be myristoylated using myristoyl chloride (1 equivalent) and diisopropylethylamine (2.5 equivalents) in di-

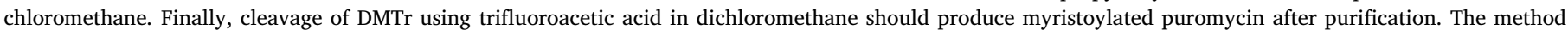

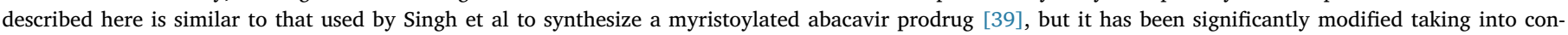

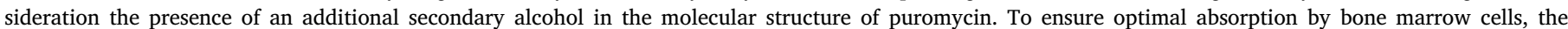

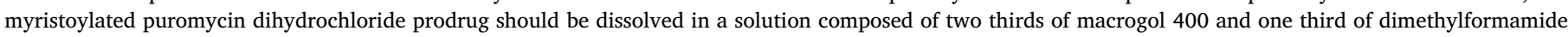
with a concentration of $16 \mathrm{mg} / \mathrm{ml}$. One dose of this solution should be diluted in eleven doses of $0.9 \%$ sodium chloride before injection. (See busulfan).

is able to express quintuplex gRNAs plus the humanized SpCas9 and the puromycin resistance gene linked by $\mathrm{T} 2 \mathrm{~A}$, or an equivalent vector.

5. Then a few weeks later, perform in vivo positive selection; give the patient a combined intravenous injection of myristoylated puromycin prodrug and tariquidar with a dosing regimen of respectively 1.2 and $4 \mathrm{mg} / \mathrm{kg} /$ day during three series of three days with a seven-day spacing between each series (Dosage can be adjusted if necessary).

\section{Testing the hypothesis}

My hypothesis is relatively simple to test. First of all, my vector should undergo a series of in vitro tests to check its stability and efficiency and if possible improve it. Then, if my reasoning is correct and my vector optimal, the implementation of a clinical trial based on the protocol that I have just described (and that I named Grenoble protocol in tribute to my city) would be more than justified. Of course, such a trial should respect all ethical standards and be performed by qualified personnel. For my hypothesis to be validated, the HIV RNA should become undetectable and remain so throughout the rest of the life of the patient, which would be the first functional cure in humans.

Furthermore, although puromycin is a particularly potent cytotoxic agent, the mortality rate caused by the positive selection should be strictly below the $5 \%$ threshold, which is perfectly acceptable. However, due to the choice of gRNAs, the Grenoble protocol would be effective only on patients infected by HIV-1 M group, which still affects $96 \%$ of the total infected population, but it is possible to adapt my strategy to other HIV subtypes by using the same approach.

Besides, I estimate the total cost of implementing this experimental treatment at several hundred thousand dollars per patient, although a large-scale implementation should make the price affordable.

\section{Implications of the hypothesis}

The Grenoble protocol could have a major impact on HIV-infected people. Indeed, in case of confirmation by a clinical trial, AIDS should be reclassified as a curable disease.

Taken separately, none of the elements constituting the Grenoble protocol are really novel, but once combined, they form a therapeutic strategy that could become a reference treatment against AIDS, although, for the moment, all this is only at the stage of hypothesis and theory.

\section{Declaration of Competing Interest}

The authors declare that they have no known competing financial interests or personal relationships that could have appeared to influence the work reported in this paper.

\section{Acknowledgements}

I would like to personally thank Professor Doctor Ben Berkhout for agreeing to review my work and giving me some technical advice.

\section{References}

[1] Barre-Sinoussi $\mathrm{F}$, et al. Isolation of a T-lymphotropic retrovirus from a patient at risk for acquired immune deficiencysyndrome (AIDS). Science 1983;220:868-71. https://doi.org/10.1126/science.6189183.

[2] Chun TW, et al. Presence of an inducible HIV-1 latent reservoir during highly active antiretroviral therapy. Proc Natl Acad Sci 1997;94:13193-7. https://doi.org/10. 1073/pnas.94.24.13193.

[3] Lorenzo-Redondo R, et al. Persistent HIV-1 replication maintains the tissue reservoir during therapy. Nature 2016;530:51-6. https://doi.org/10.1038/nature16933.

[4] Deeks SG, et al. International AIDS Society global scientific strategy: towards an HIV cure 2016. Nat Med 2016;22:839-50. https://doi.org/10.1038/nm.4108.

[5] Hütter G, et al. Long-term control of HIV by CCR5 Delta32/Delta32 stem-cell transplantation. New Engl J Med 2009;360:692-8. https://doi.org/10.1056/ NEJMoa0802905.

[6] Gupta RK, et al. HIV-1 remission following CCR5 $\Delta 32 / \Delta 32$ haematopoietic stem-cell transplantation. Nature 2019;568:244-8. https://doi.org/10.1038/s41586-0191027-4.

[7] Jinek M, et al. A programmable dual-RNA-guided DNA endonuclease in adaptive bacterial immunity. Science 2012;337:816-21. https://doi.org/10.1126/science. 1225829.

[8] Mali P, et al. RNA-guided human genome engineering via Cas9. Science 2013;339:823-6. https://doi.org/10.1126/science.1232033.

[9] Ran FA, et al. Genome engineering using the CRISPR-Cas9 system. Nat Protocols 2013;8:2281-308. https://doi.org/10.1038/nprot.2013.143.

[10] Cho SW, Kim S, Kim JM, Kim JS. Targeted genome engineering in human cells with the Cas9 RNA-guided endonuclease. Nat Biotechnol 2013;31:230-2. https://doi. org/10.1038/nbt. 2507 .

[11] Shalem O, et al. Genome-scale CRISPR-Cas9 knockout screening in human cells. Science 2014;343:84-7. https://doi.org/10.1126/science.1247005.

[12] Hsu P, Lander E, Zhang F. Development and applications of CRISPR-Cas9 for genome engineering. Cell 2014;157:1262-78. https://doi.org/10.1016/j.cell.2014. 05.010 .

[13] Hsu PD, et al. DNA targeting specificity of RNA-guided Cas9 nucleases. Nat Biotechnol 2013;31:827-32. https://doi.org/10.1038/nbt.2647.

[14] Ran F, et al. Double nicking by RNA-guided CRISPR Cas9 for enhanced genome editing specificity. Cell 2013;154:1380-9. https://doi.org/10.1016/j.cell.2013.08. 021.

[15] Wang T, Wei JJ, Sabatini DM, Lander ES. Genetic screens in human cells using the CRISPR-Cas9 system. Science 2014;343:80-4. https://doi.org/10.1126/science. 1246981.

[16] Gasiunas G, Barrangou R, Horvath P, Siksnys V. Cas9-crRNA ribonucleoprotein 
complex mediates specific DNA cleavage for adaptive immunity in bacteria. Proc Natl Acad Sci 2012;109:E2579-86. https://doi.org/10.1073/pnas.1208507109.

[17] Mali P, et al. CAS9 transcriptional activators for target specificity screening and paired nickases for cooperative genome engineering. Nat Biotechnol 2013;31:833-8. https://doi.org/10.1038/nbt.2675.

[18] Ran FA, et al. In vivo genome editing using Staphylococcus aureus Cas9. Nature 2015;520:186-91. https://doi.org/10.1038/nature14299.

[19] Ebina H, Misawa N, Kanemura Y, Koyanagi Y. Harnessing the CRISPR/Cas9 system to disrupt latent HIV-1 provirus. Sci Rep 2013;3:2510. https://doi.org/10.1038/ srep0251.

[20] Liao HK, et al. Use of the CRISPR/Cas9 system as an intracellular defense against HIV-1 infection in human cells. Nat Commun 2015;6:6413. https://doi.org/10. 1038/ncomms7413.

[21] Wang Z, et al. CRISPR/Cas9-derived mutations both inhibit HIV-1 replication and accelerate viral escape. Cell Rep 2016;15:481-9. https://doi.org/10.1016/j.celrep. 2016.03.042.

[22] $\mathrm{Hu}$ W, et al. RNA-directed gene editing specifically eradicates latent and prevents new HIV-1 infection. Proc Natl Acad Sci 2014;111:11461-6. https://doi.org/10. 1073/pnas.1405186111.

[23] Zhu W, et al. The CRISPR/Cas9 system inactivates latent HIV-1 proviral DNA. Retrovirology 2015;12:22. https://doi.org/10.1186/s12977-015-0150-z.

[24] Kaminski R, et al. Elimination of HIV-1 genomes from human T-lymphoid cells by CRISPR/Cas9 gene editing. Sci Rep 2016;6:22555. https://doi.org/10.1038/ srep22555.

[25] Kaminski R, et al. Excision of HIV-1 DNA by gene editing: a proof-of-concept in vivo study. Gene Ther 2016;23:690-5. https://doi.org/10.1038/gt.2016.41.

[26] Yin C, et al. In vivo excision of HIV-1 provirus by saCas9 and multiplex single-guide RNAs in animal models. Mol Ther 2017;25:1168-86. https://doi.org/10.1016/j. ymthe.2017.03.012.

[27] Ophinni Y, Inoue M, Kotak T, Kameoka M. CRISPR/Cas9 system targeting reg ulatory genes of HIV-1 inhibits viral replication in infected T-cell cultures. Sci Rep 2018;8:7784. https://doi.org/10.1038/s41598-018-26190-1.
[28] Wang G, Zhao N, Berkhout B, Das AT. A combinatorial CRISPR-Cas9 attack on HIV1 DNA extinguishes all infectious provirus in infected $\mathrm{T}$ cell cultures. Cell Rep 2016;17:2819-26. https://doi.org/10.1016/j.celrep.2016.11.057.

[29] Bella R, et al. Removal of HIV DNA by CRISPR from patient blood engrafts in humanized mice. Mol Ther Nucleic Acids 2018;12:275-82. https://doi.org/10.1016/j. omtn.2018.05.021.

[30] Dash PK, et al. Sequential LASER ART and CRISPR treatments eliminate HIV-1 in a subset of infected humanized mice. Nat Commun 2019;10:2753. https://doi.org/ 10.1038/s41467-019-10366-y.

[31] Robertson DL, Hahn BH, Sharp PM. Recombination in AIDS viruses. J Mol Evol 1995;40:249-59. https://doi.org/10.1007/BF00163230.

[32] Rambaut A, Posada D, Crandall KA, Holmes EC. The causes and consequences of HIV evolution. Nat Rev Genet 2004;5:52-61. https://doi.org/10.1038/s41467-01910366-y.

[33] Perelson AS, Ribeiro RM. Estimating drug efficacy and viral dynamic parameters: HIV and HCV. Stat Medicine 2008;27:4647-57. https://doi.org/10.1002/sim.3116.

[34] Locatelli A. pLV. 2019. https://doi.org/10.6084/m9.figshare.9642281.v1.

[35] Amirache F, et al. Mystery solved: VSV-G-LVs do not allow efficient gene transfer into unstimulated T cells, B cells, and HSCs because they lack the LDL receptor. Blood 2014;123:1422-4. https://doi.org/10.1182/blood-2013-11-540641.

[36] Alberts B, et al. Molecular Biology of the Cell. 4th edition. New York: Garland Science; 2002. Table 22-1, Blood Cells. https://www.ncbi.nlm.nih.gov/books/ NBK26919/table/A4143/.

[37] Abkowitz JL, Catlin SN, McCallie MT, Guttorp P. Evidence that the number of hematopoietic stem cells per animal is conserved in mammals. Blood 2002;100:2665-7. https://doi.org/10.1182/blood-2002-03-0822.

[38] Bhukhai K, et al. Ex vivo selection of transduced hematopoietic stem cells for gene therapy of $\beta$-hemoglobinopathies. Mol Ther 2018;26:480-95. https://doi.org/10 1016/j.ymthe.2017.10.015.

[39] Singh D, et al. Development and characterization of a long-acting nanoformulated abacavir prodrug. Nanomedicine 2016;11:1913-27. https://doi.org/10.2217/nnm2016-0164 